\title{
SMALL-ANGLE SCATTERING OF SYNCHROTRON RADIATION FOR STUDYING SOLID STATE AND BIOLOGICAL SYSTEMS
}

\author{
P. Fratzl \\ Institut für Festkörperphysik der Universität Wien \\ Strudlhofgasse 4, 1090 Wien, Austria
}

\begin{abstract}
The principles of small-angle X-ray scattering are briefly reviewed with emphasis on the particular advantages of the use of synchrotron radiation. The application of the technique is illustrated by several examples concerning precipitates in alloys, internal oxidation, porous materials, as well as the structure of bone.
\end{abstract}

PACS numbers: $61.10 . \mathrm{Lx}, 64.75 .+\mathrm{g}, 81.30 . \mathrm{Mh}, 87.22 . \mathrm{Bt}$

\section{Introduction}

Small-angle scattering of X-rays (or neutrons) allows the study of structures with sizes from $\approx 1$ to $\approx 100 \mathrm{~nm}$, and the applications range from biology to materials science. Traditionally, the technique has been applied to structural analysis in two very different ways. The first, crystallographic small-angle diffraction, is used to study the periodic arrangement of atoms, molecules, or groups of molecules. In this case one has to interpret sharp Bragg peaks occurring at small scattering angles. This technique, which relates to crystallography, shall not be discussed here. The second way, which is the subject of this paper, consists in the interpretation of the broad, diffuse small-angle scattering containing information on size, shape, and orientation of inhomogeneities (like precipitates, pores, macromolecules in solution, etc.) with sizes ranging from $\approx 1$ to $\approx 100 \mathrm{~nm}$. Such kind of information may, in principle, also be obtained by electron microscopy. The advantage of small-angle scattering is the fact that the obtained parameters (size, shape, orientation, etc.) represent average values over macroscopic sample regions, whereas the advantage of electron microscopy is the possibility to image individual objects.

Small-angle scattering has been introduced mainly by Guinier [1] and by Kratky and Porod [2]. The principles of the method are reviewed in several textbooks [3-5]. In recent years, the availability of strong X-ray source as well as position-sensitive detectors has increased the potential of the technique and with the use of synchrotron radiation new applications (like, e.g., anomalous scattering, time-resolved studies, three-dimensional reconstruction of anisotropic scattering) 
became possible. It is the aim of the present paper to briefly review the principles of small-angle scattering with emphasis on these new possibilities using synchrotron radiation. This will be complemented by several examples of recent investigations. These examples are chosen with respect to the particular interest of the author and are not representative for the whole spectrum of possible applications. For further applications the reader might refer to recent reviews on the subject (biological systems [6-9], polymer systems [10-12], anorganic material [13-16]).

\section{Principles of small-angle scattering}

\subsection{Data collection}

To show the typical setup for a small-angle scattering experiment, the beamline JUSIFA [17] at the synchrotron DESY in Hamburg is shown schematically in Fig. 1. From the white synchrotron radiation, a monochromatic beam is selected by a double-crystal monochromator. The beam is then collimated by a series of pinholes and its intensity measured by a monitor counter before it hits the sample. The X-ray intensity scattered in the sample under small angles $\theta$ is finally collected in a two-dimensional position-sensitive detector (Fig. 1).

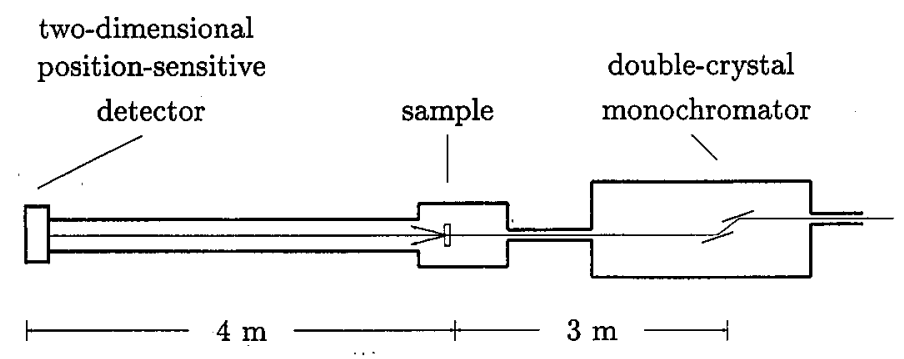

Fig. 1. Sketch of a typical small-angle scattering beamline [17].

To account for the parasitic scattering from the pinholes, the measured X-ray intensity is corrected in the following way [12]:

$$
I=(T-D) / t-(P-D),
$$

where $T$ is the total intensity, $P$ - the parasitic pinhole scattering (measured without sample) and $D$ - the dark current of the detector (measured without $\mathrm{X}$-ray beam). $t$ is the transmission of the sample for the $\mathrm{X}$-ray beam. Usually, the $\mathrm{X}$-ray intensity $I$ is written as a function of the scattering vector $k$, the length of $\boldsymbol{k}$ given by

$$
|k|=k=4 \pi / \lambda \sin (\theta / 2),
$$

where $\lambda$ is the wavelength of the incident X-ray beam. The direction of $k$ is defined by the difference between the incident and the diffracted beam. 


\subsection{General equations}

The scattered X-ray intensity may be written in general [3-5]:

$$
\left.\left|(k)=I_{0}\right| \sum f_{j} \exp \left(\mathrm{i} k \cdot r_{j}\right)\right|^{2},
$$

where $f_{j}$ is the scattering factor of the atom at position $r_{j}$ and $I_{0}-$ a factor depending on the experimental setup. For an $\mathrm{X}$-ray energy far enough from any absorption edge the scattering factor of the atom is approximately equal to its number of electrons. In an approximation frequently used in small-angle scattering experiments, the two-phase model [2-5], the sample is considered to be made of two phases (a matrix and inclusions) with different average scattering factor and separated by sharp interfaces. In this case, the diffuse intensity at small angles writes approximately

$$
\left.\left|(k)=J_{0}\left(f_{A}-f_{B}\right)^{2}\right| \int_{A} \exp (\mathrm{i} k \cdot r) \mathrm{d}^{3} r\right|^{2},
$$

where $f_{A}$ and $f_{B}$ are the average scattering factors of the phases $A$ and $B$, and $J_{0}$ is a constant. $\left(f_{A}-f_{B}\right)^{2}$ is sometimes called the "contrast" between the two phases.

Equation (4) can be used to calculate the scattering pattern for a given shape of the inclusion $A$. For example, calling $k_{x}, k_{y}, k_{z}$ the components of the vector $k$ into the three directions of space, one obtains for a parallelepiped of side-lengths $a, b, c[3-5]$ :

$$
I(k)=I(0) A^{2}\left(A k_{x}\right) A^{2}\left(b k_{y}\right) A^{2}\left(c k_{z}\right) \text {. with } A(x)=(2 / x) \sin (x / 2),
$$

and for a cylinder with radius $r$ and height $h$ (directed along the $z$-axis) [3-5]:

$$
I(k)=I(0) A^{2}\left(h k_{z}\right) B^{2}\left(r \sqrt{k_{x}^{2}+k_{y}^{2}}\right) \quad \text { with } B(x)=(2 / x) J_{1}(x),
$$

$J_{1}(x)$ being the Bessel function of the first kind. The scattering of a sphere of radius $r$ is, of course, isotropic and given by [3-5]:

$$
I(k)=I(k)=I(0) C^{2}(r k) \text { with } C(x)=\left(3 / x^{3}\right)[\sin (x)-x \cos (x)] .
$$

\subsection{Isotropic systems}

In isotropic liquids, in polycrystals or when the inclusions are randomly oriented the scattering intensity $I(k)$ has spherical symmetry and does only depend on the value $k$ of the vector $k$. In this situation several general equations can be given for the two-phase model.

The first one is the Guinier law [3-5] derived under the assumption of medium $B$ containing isolated inclusions $A$ with a distance between the inclusions much larger than their size. In this case the intensity is approximately Gaussian at small $k$ and can be written [3]:

$$
I(k)=I(0) \exp \left(-k^{2} R^{2} / 3\right) \quad(\text { small } k),
$$

where $R$ is the radius of gyration of the inclusions. For instance, if the inclusions are spherical with a radius $r$, then $R^{2}=(3 / 5) r^{2}$. Figure 2 shows the small-angle 


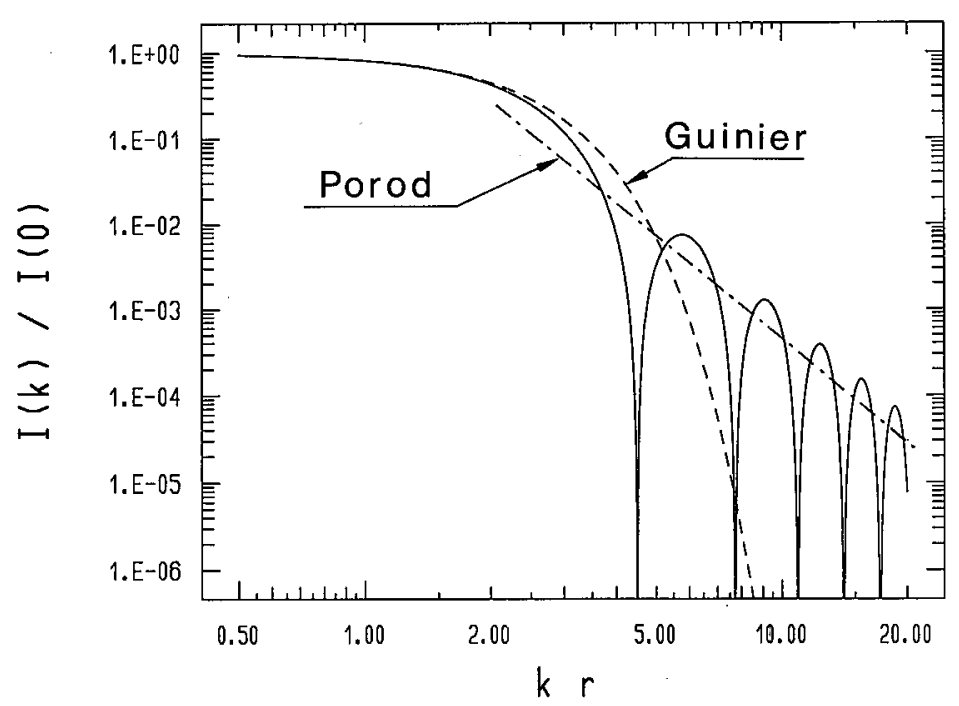

Fig. 2. Small-angle scattering from a sphere of radius $r$ (Eq. (7)). The broken line corresponds to the Guinier approximation (Eq. (8)) and the dashed-dotted line to Porod's law (Eq. (10)).

scattering from spherical inclusions (Eq. (7)). The Guinier law is widely used for the determination of the average size of inclusions.

Where the distance between precipitates is not large enough, interference effects between precipitates cannot be neglected and the Guinier law is not valid anymore. Nevertheless, an evaluation using Eq. (8) in many cases still gives the right order of magnitude for the average inclusion size [18]. Although a general equation cannot be given for the scattering from densely packed inclusions, there are expressions for several special cases. One such expression will be discussed later in Sec. 3.2. In the case where identical, randomly oriented inclusions are distributed in a matrix, the intensity writes [3-5]:

$$
I(k)=K_{0} F(k)\left|\sum \exp \left(\mathrm{i} k \cdot c_{j}\right)\right|^{2},
$$

where $K_{0}$ is a constant, $F(k)$ is the form factor of a single inclusion (e.g. calculated via Eq. (4) with $A$ taken to be the inclusion) and $c_{j}$ are the center positions of the inclusions.

In the cases where (isolated) inclusions have some variation in size, this polydispersity must be taken into account by averaging the form factor $F(k)$ over the different inclusion sizes. Equation (9) then, in general, does not remain valid. In the case of spherical inclusions (see Fig. 2), the effect of averaging over different radii has the effect of gradually wiping out the oscillations of the form factor. This is shown later for an example of spherical precipitates in a metal alloy. For system containing only spherical inclusions (for instance colloidal dispersions [19]), mathematical procedures are available to extract the size distribution of the spheres from small-angle scattering data $[20,21]$. 
Another general equation is Porod's law [3-5], which does not require the hypothesis of isolated inclusions. Starting from Eq. (4), the scattering intensity at large values of $k$ becomes [2-5]:

$$
I(k)=J_{0}\left(f_{A}-f_{B}\right)^{2} 2 \pi S / k^{4} \quad(\text { large } k),
$$

where $S$ is the total surface in between the two phases $A$ and $B$. An example for spherical inclusions is shown in Fig. 2. Porod's law may be used to determine the internal surface of, e.g., porous material or catalysts [15].

In cases where a smooth internal surface cannot be defined because the structure has a fractal character [15], small-angle scattering is able to give the fractal dimension of the system. The essential property of fractal system is that they appear identical on any scale of magnification at least between a lower and a larger limit of scale. Physically, such a structure can be expected, e.g., for rough surfaces or randomly porous material. A recent review of small-angle scattering from fractal systems can be found in [15]. The only property to be mentioned here is that the self-similarity of the fractal structure leads to a power-law behaviour of the intensity in the form

$$
I(k)=H_{0} k^{-\alpha} \text {, }
$$

where $H_{0}$ is a constant, $\alpha=D_{\mathrm{M}}$ for a mass fractal with dimension $D_{\mathrm{M}}$ and $\alpha=6-D_{\mathrm{F}}$ for a surface fractal with dimension $D_{\mathrm{F}}$. Note that for a smooth internal surface $D_{\mathrm{F}}=2$ and Eq. (11) reduces to Porod's law (10).

\subsection{Anisolropic systems}

Anisotropic inclusions produce anisotropic small-angle scattering as illustrated schematically in Fig. 3. Due to the fact that the shape of any object relates to

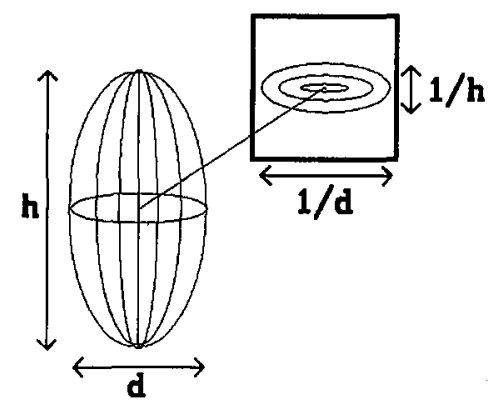

Fig. 3. Schematic representation of the scattering from a non-spherical object. The square symbolizes a two-dimensional detector and the lines on the detector are isointensity contours.

its scattering pattern by a Fourier transformation (Eq. (4)), the dimensions of the object in Fig. 3 correspond roughly to the inverse dimensions of the scattering pattern. Therefore, if non-spherical inclusions are distributed with a well-defined orientation inside a matrix, their shape size and orientation can be deduced from their 
three-dimensional scattering pattern. Each measurement with the two-dimensional detector is just a planar section through this three-dimensional scattering pattern which can, therefore, be reconstructed by a series of measurements with various angles between the sample and the incoming X-ray beam.

After Fig. 3, the typical scattering pattern to be expected from oriented plane-shaped inclusions (e.g. precipitates in a single crystal) will be a streak in reciprocal space. To illustrate this, plate-like inclusions (approximated by cylinders with diameter $d$ and height $h<d$ ) are supposed to be distributed on (111)-planes of a cubic crystal. The scattering is then given by Eq. (6), where the $z$-direction is chosen to be the [111]-direction of the crystal. As in a cubic crystal there are four [111]-directions, the total scattering will be the sum of four terms calculated by Eq. (6), each with the $z$-direction parallel to one of the [111]-directions. In the ex-

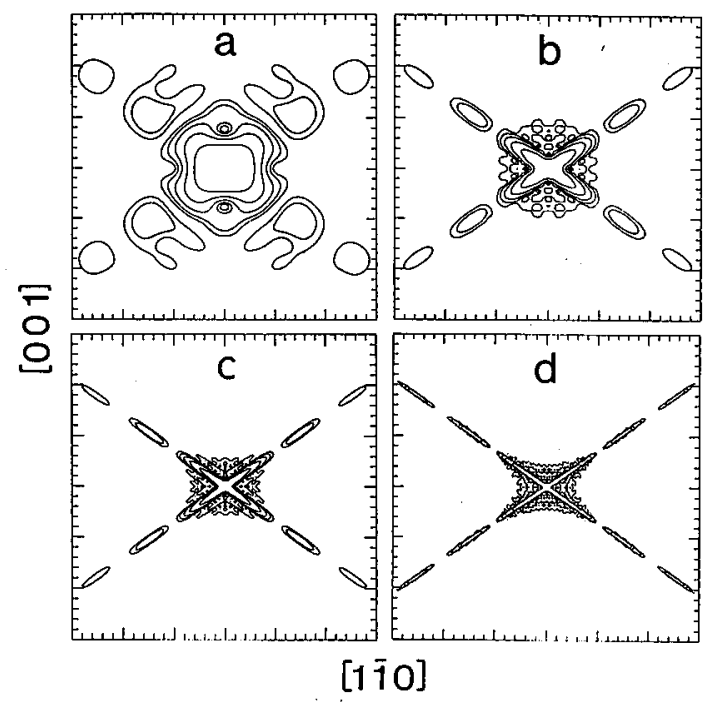

Fig. 4. Calculated intensity (using Eq. (6)) in the (110)-plane of cubic crystal containing plate-like inclusions lying on (111)-plates. The lines are isointensity contours and represent the logarithm of scattering intensity. The aspect ratio of the cylindrical plates (i.e. the ratio height to diameter) is 0.5 in (a), 0.2 in (b), 0.1 in (c) and 0.05 in (d).

ample shown in Fig. 4, the crystal is oriented with its (110)-plane perpendicular to the incoming X-ray beam. The X-ray pattern was calculated for four different aspect ratios $h / d$. The scattering patterns are elongated into the two [111]-directions contained in this (110)-plane. One can clearly see that with decreasing aspect ratio $h / d$, the streaks become sharper. In fact, the length of the streaks relates to $1 / h$ and the width to $1 / d$. Anisotropic spectra from single crystals can, therefore, be used to determine shape, size, and orientation of the inclusions.

Finally, one can see in Fig. 4 oscillations of the X-ray intensity along the direction of the streaks. These have a similar origin as the oscillations in Fig. 2 and are wiped out as soon as the inclusions have some distribution in size. 


\subsection{Anomalous scaltering}

A particular advantage of the synchrotron X-ray beam is the fact that the energy $E$ of the incoming beam can be varied continuously. This allows to take advantage of the anomalous dispersion [22-26] of the atomic scattering factor $f$ (entering Eq. (3)) close to absorption edges. Usually one writes

$$
f=Z+f^{\prime}(E)+\mathrm{i} f^{\prime \prime}(E),
$$

where $Z$ is the atomic number and the dispersion terms $f^{\prime}$ and $f^{\prime \prime}$ are tabulated as functions of $E$ [27].

In the two-phase model (Eq. (4)), the variation of the scattering factors changes the contrast $\left(f_{A}-f_{B}\right)^{2}$, which may be either enhanced or reduced by the right choice of $E$. The particular advantage of contrast variation is the possibility to separate the different contributions in systems that contain more than two components like, e.g., multicomponent alloys [25, 26] or complex molecules [22, 23], by measuring the small-angle scattering at several X-ray energies $E$. An example of a binary alloy with surface defects (= the third component) is shown later in Sec. 3.1.

\section{Examples}

\subsection{Spherical precipitates in dilute $\mathrm{Cu}-\mathrm{Fe}$}

The solubility of iron copper decreases strongly with temperature. When a sample of $\mathrm{Cu}_{1-x} \mathrm{Fe}_{x}$ (with $x=1$ at\%) is quenched from $1000^{\circ} \mathrm{C}$ and subsequently annealed at temperatures between 300 and $700^{\circ} \mathrm{C}$, one observes the formation of almost spherical $\gamma$-precipitates, which can be studied by small-angle scattering $[28,29]$. In this case, a large contribution from crystal defects (e.g. at the macroscopic surface) is superimposed on the X-ray scattering from the Fe-precipitates. These two contributions can be separated using anomalous scattering. Indeed, the contrast (see Eq. (4)) between Cu-matrix and Fe-precipitates is given by $\left(f_{\mathrm{Cu}}-f_{\mathrm{Fe}}\right)^{2}$ and the contrast between matrix and crystal defects by $\left(x f_{\mathrm{Cu}}+(1-x) f_{\mathrm{Fe}}\right)^{2}$. The first varies strongly in the vicinity of the $\mathrm{Fe} K$-edge, whereas the second stays practically constant. Figure 5 shows the X-ray intensity $I(k)$ measured at two energies $E$ of the X-ray beam, one close to and one well below the Fe $K$-edge. At small $k$, where the scattering from crystal defects dominates, the spectrum is practically independent of $E$, and at larger $k$, where the scattering from the precipitates dominates, there is a marked change with $E$ (Fig. 5). After removal of the scattering from crystal defects, the small-angle scattering is as plotted in Fig. 6 (reproduced from [29]). The broken line in Fig. 6 shows the spectrum expected for spherical precipitates (see Fig. 2) and the full line is obtained by averaging the broken line using a physically reasonable size distribution [29] of the precipitates. As discussed in Sec. 2.3 the effect of polydispersity of the spheres is to wipe out the oscillations of the scattering function (see Fig. 6). Moreover, the amount of precipitates is small enough that interference effects between precipitates can be neglected in this case. 


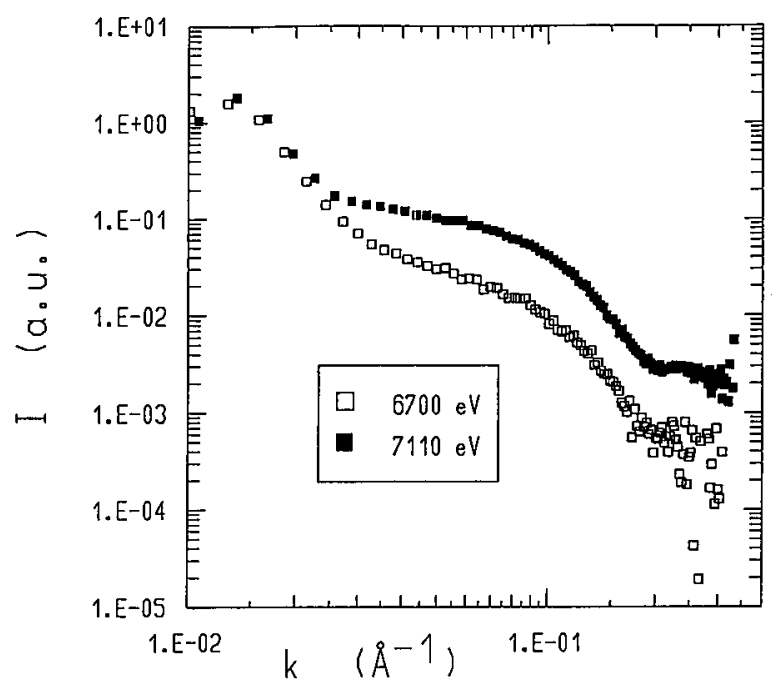

Fig. 5. X-ray scattering from a dilute $\mathrm{Cu}-\mathrm{Fe}$ alloy at two different energies of the $\mathrm{X}$-ray beam (from [28]).

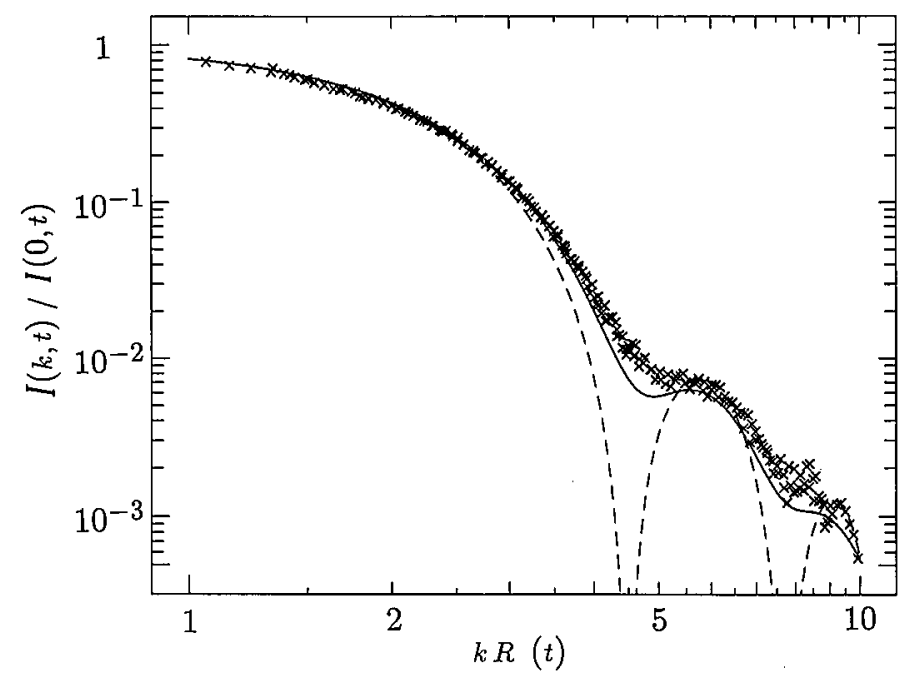

Fig. 6. Scattering intensity from a dilute $\mathrm{Cu}-\mathrm{Fe}$ alloy corrected for contributions from defects of the crystal structure (from [29]). $R(t)$ is the Guinier radius defined by Eq. (8).

\subsection{Kinetics of phase separation}

Small-angle scattering is a widely used method for the study of kinetics of phase separation (like in alloys [14] or polymer systems [10]). One of the reasons is that for binary systems, Eq. (3) is just the Fourier transform of the pair-correlation function, which is a quantity well-accessible to theoretical treatments [30-32]. 
Therefore, the evolution of $I(k)$ during a phase separation process can be theoretically predicted under certain assumptions and directly compared to experiment data.

The first prediction of this kind was Cahn's theory of spinodal decomposition [33], designed to describe the early stages of a phase separation process. This theory is based on a modified diffusion equation known as Cahn-Hillard equation [34]:

$$
\mathrm{d} u / \mathrm{d} t=M \nabla^{2}\left(f^{\prime}(u)-K \nabla^{2} u\right),
$$

where $u$ is the concentration of $B$ atoms in an $A-B$ mixture, $f^{\prime}$ is the derivative of the free energy density and $M$ and $K$ are constants. Noting that the small-angle scattering intensity $I$ is proportional to $|\hat{u}|^{2}$ ( $\hat{u}$ being the Fourier transform of $u$ ), the solutions of this equation may be used to predict the time-evolution of $I$. Indeed, various approximate solutions of Eq. (13) have been obtained analytically $[33,35,36]$ and numerically $[37,38]$ and the results. were compared to experiment data (for a recent review see [32]). The most prominent feature of $I(k)$ is the appearance of an interference maximum at a position $k_{\mathrm{m}}(t)$ which shifts with time.This is shown in Fig. 7a for the model system $\mathrm{Al}-\mathrm{Zn}-\mathrm{Mg}$ (redrawn from [39]).
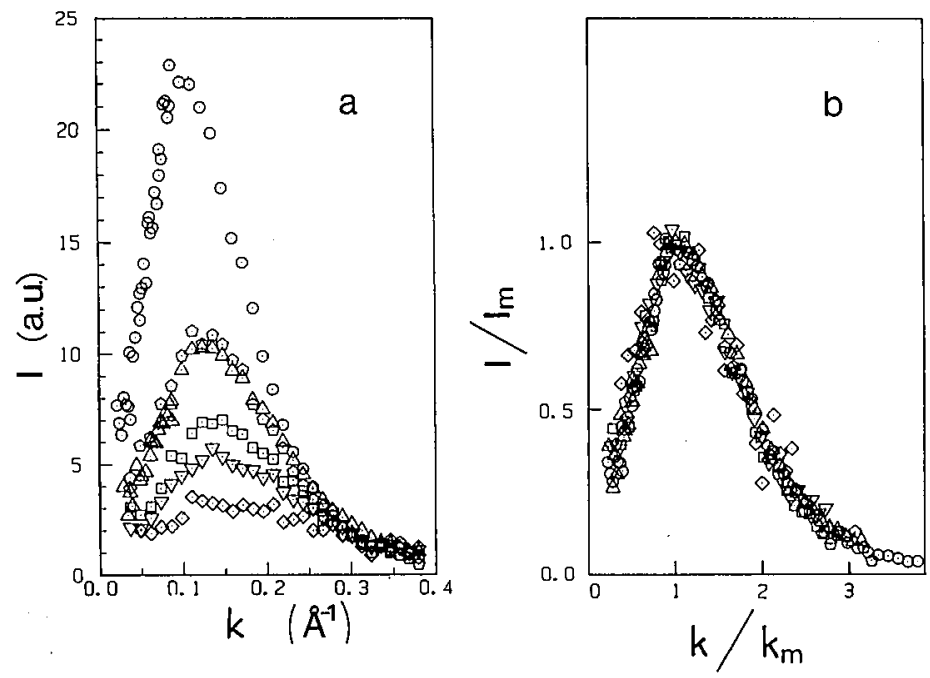

Fig. 7. Small-angle scattering intensity from $\mathrm{Al}-2.4$ at\% $\mathrm{Zn}-1.3$ at\% $\mathrm{Mg}$ annealed at room temperature for 6 different times between 24 hours and 1 year (from [39]). $I_{\mathrm{m}}$ and $k_{\mathrm{m}}$ are defined in Eq. (14).

In the late stages of phase separation, one frequently observes a time-scaling behavior of the X-ray intensity at time $t, I(k, t)$, in the form [40, 18, 30-32]:

$$
I(k, t)=I_{\mathrm{m}}(t) F\left(k / k_{\mathrm{m}}(t)\right),
$$

where $F$ is a function independent of time and $k_{\mathrm{m}}(t)$ and $I_{\mathrm{m}}(t)=I\left(k_{\mathrm{m}}(t), t\right)$ are time-dependent constants. This time-scaling property is observed for many 
systems and in particular also for $\mathrm{Al}-\mathrm{Zn}-\mathrm{Mg}[41,42]$. Beside the time evolution of $I$ for the Al-Zn-Mg system (Fig. 7a), the same data were plotted (Fig. 7b) on rescaled axes $k / k_{\mathrm{m}}(t)$ and $I(k, t) / I_{\mathrm{m}}(t)$. All spectra fall onto the same graph which represents the scaling function $F(x)$. The time-scaling behavior (Eq. (14)), therefore, means that the system looks the same at different times $t$ when the magnification is changed by a factor $k_{\mathrm{m}}(t)$.

The maximum in $I(k)$ (Fig. 7) clearly shows that interference effects between precipitates cannot be neglected, in general, for systems undergoing phase separation. But Eq. (9), which accounts for interference effects of identical inclusions, cannot be used, because the precipitates have no reason to be equal in size throughout the sample. Nevertheless, it is possible, using heuristic arguments, to give an analytic expression for the scaling function $F(x)$ which accounts for the interference effects in this special case [43, 44]:

$$
F(x) \approx \frac{a b x^{4}}{\left(x^{4}+c\right)\left[b+\left(x^{2}-1+d\right)^{2}\right]},
$$

where the constants $a, b, c, d$ are explicitly defined and depend only on the phase diagram [43]. Measurements of $F(x)$ for different $\mathrm{Al}_{1-x} \mathrm{Ag}_{x}$ alloys [45] are compared to Eq. (15) in Fig. 8. Equation (15) is, however, only valid when the reduction of

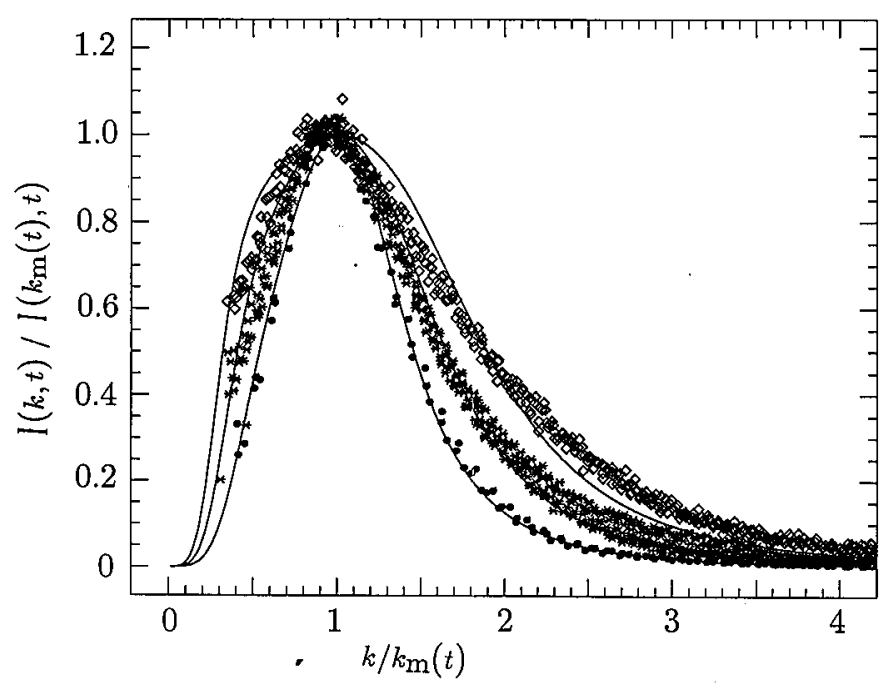

Fig. 8. Scaling curves $F(x)$ (defined in Eq. (14)) for precipitates in $\mathrm{Al}_{1-x} \mathrm{Ag}_{x}$, with $x=5$ at\% (circles), $x=2$ at\% (stars) and $x=1$ at\% (squares) (from [29]). The full lines are calculated with Eq. (15).

surface between matrix and precipitates is the driving force for precipitate coarsening [44]. When, e.g., elastic interaction between precipitates is important, then Eq. (15) is no longer valid [46]. 


\subsection{Internal oxidation}

The formation of internal oxide precipitates can occur, e.g., in pure silicon, due to a small amount of dissolved oxygen. This has been shown by (neutron) small-angle scattering to give cushion-shaped precipitates [47]. This evidence was obtained by interpreting spectra similar to Fig. 4 obtained with single crystals [47].

The formation of oxide precipitates does also occur when an alloy of two elements with very different affinities to oxygen (like copper and iron) is heated up in an oxygen atmosphere. Internally oxidized single crystals of $\mathrm{Cu}-1$ at\% $\mathrm{Fe}$ were investigated by means of synchrotron small-angle scattering [48]. Figure 9 shows

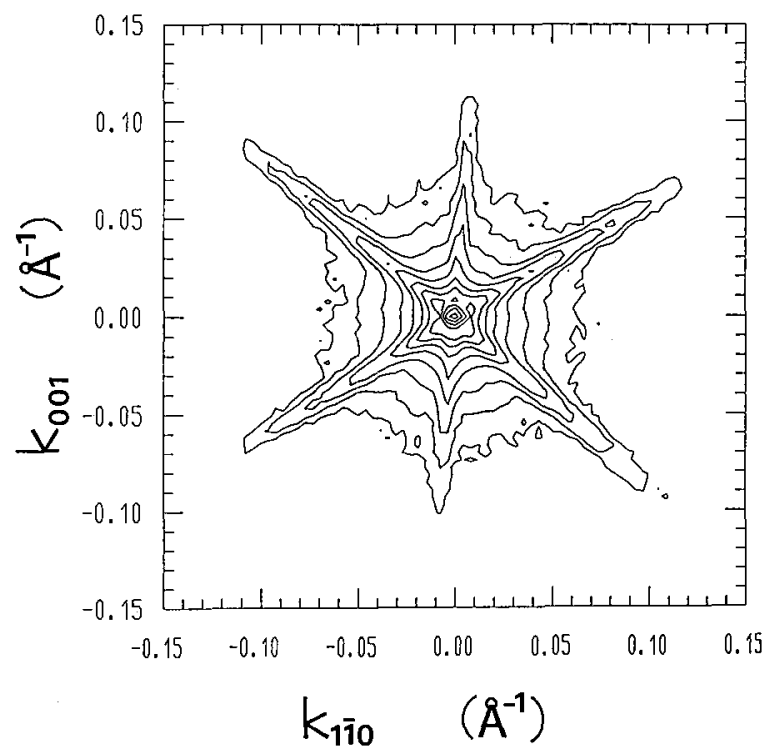

Fig. 9. X-ray scattering in the (110)-plane of a single crystal of internally oxidized $\mathrm{Cu}-1$ at\% $\mathrm{Fe}$ (from [48]).

the scattering pattern when the crystal is placed with its (110)-plane perpendicular to the X-ray beam. One observes a star-like feature with branches into [111] and [001] directions (compare to Fig. 4). Combining this result with measurements in other crystallographic planes, it can be established that the crystal contains two types of oxide plates located on (111)- and on (001)-planes of the cooper lattice. An analysis of the data in terms of Eq. (5) (analyzing the length and the thickness of 'the streaks, see Fig. 4) allows then to determine the average size of the precipitates [48].

\subsection{Porous amorphous substances}

This section briefly summarizes the results presented in [49] in order to show possible applications of Eq. (11) and of Eq. (15). For a recent review of small-angle 


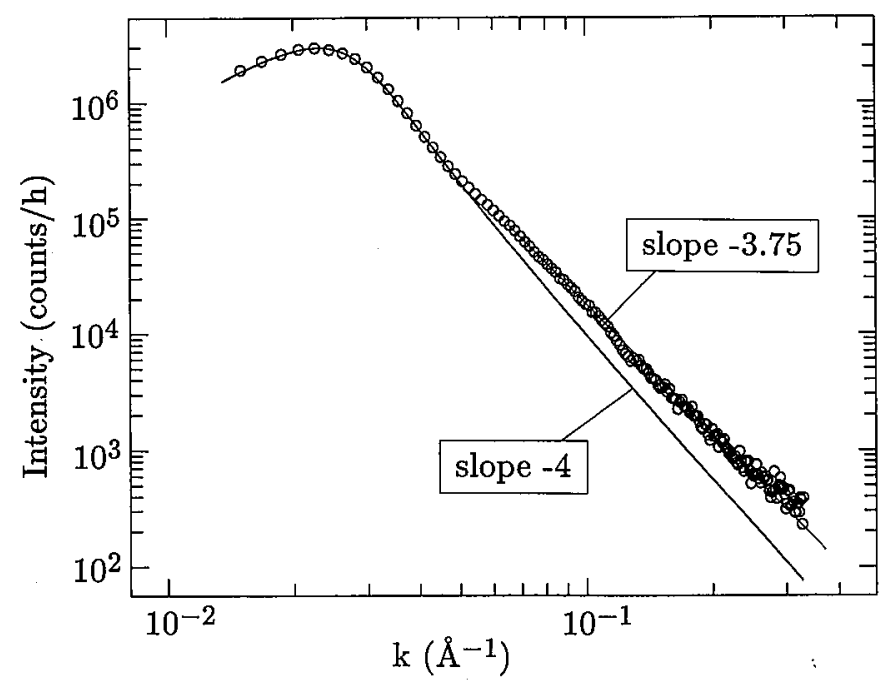

Fig. 10. Scattering intensity from porous vycor glass (from [49]).

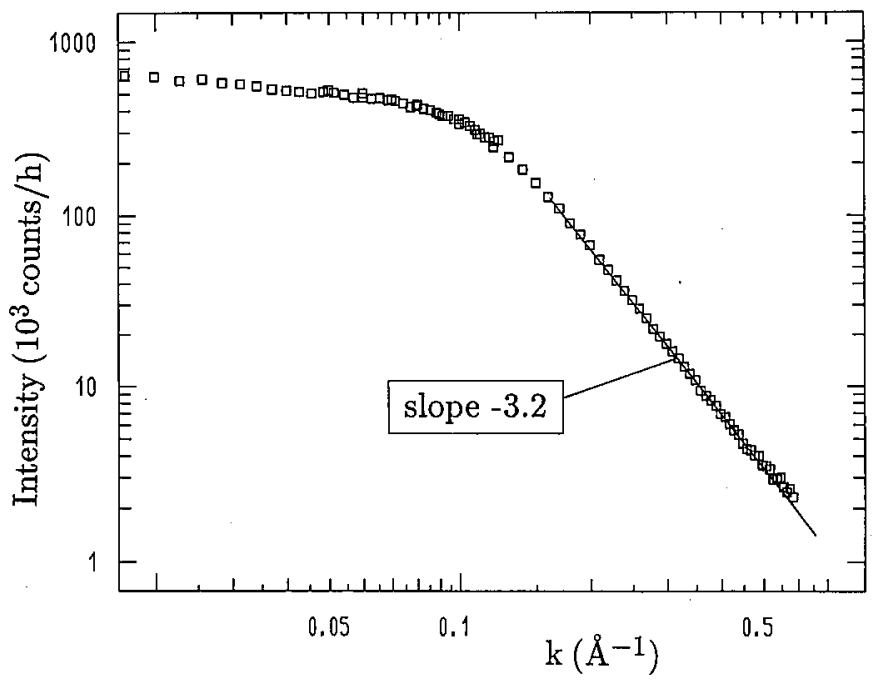

Fig. 11. Scattering intensity from glassy carbon (from [49]).

scattering from porous systems see [15]. Figures 10 and 11 show the small-angle scattering from porous vycor glass and from glassy carbon on double-logarithmic scales. The first material was produced by quenching a melt undergoing a phase separation and by subsequently leaching out one of the phases. The second material consists of entangled carbon ribbons. Both spectra exhibit a straight portion in the double-logarithmic plot (Figs. 10 and 11). According to Eq. (11), the slope of 
this straight line is just 6 minus the fractal dimension of the material considered as surface fractal. One obtains a dimension of 2.25 for the porous vycor glass (Fig. 10) and 2.8 for the glassy carbon (Fig. 11).

A very different situation can be seen in Fig. 12, which shows the scattering from amorphous silicon irradiated with heavy ions [50]. The scattering signal arises from voids growing during the irradiation process. A fit of the data with Eq. (15) (full lines in Fig. 12) allowed the determination of the total volume of the voids, which compared well to the macroscopic density $[49,50]$.

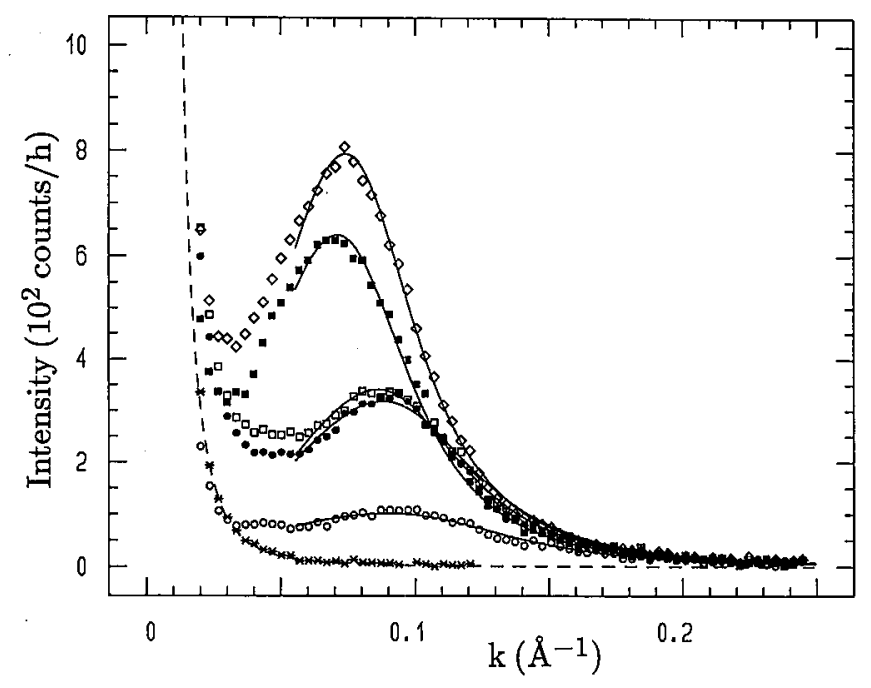

Fig. 12. Scattering intensity from amorphous silicon irradiated with heavy ions (from [50]). The increase in the maximum of the intensity curve corresponds to an increase in the irradiation influence.

\subsection{Mineral crystals in bone}

Bone is a composite material which is made essentially of long organic fibers - collagen - and calcium-phosphate mineral embedded in the collagen fibrils. As the mineral crystals are very small (a few nanometers thick), small-angle scattering is well-suited to study the size, shape and arrangement of these crystals in bone $[51,52,15]$. The protocollagen molecules are very long triple helices grouped together in fibrils. A collagen fibril containing mineral crystals is shown schematically in Fig. 13. Figure 14 shows the scattering from mineralized turkey leg tendon (reproduced from [52]), which is a bone-like tissue where the collagen fibrils are highly oriented. In Fig. 14 one recognizes a series of maxima on a vertical line due to the periodic arrangement of the molecules in the collagen fibrils (see Fig. 13). In the plane normal to this direction (the horizontal in the picture) there is a strong small-angle scattering signal (Fig. 14) from the elongated mineral crystals (Fig. 13). The data in Fig. 14 therefore allow the determination of the thickness 


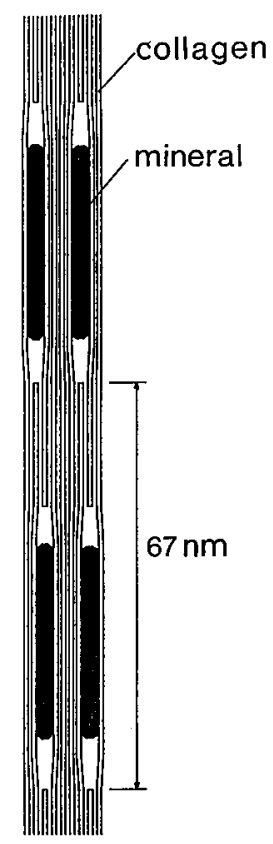

\section{mineralized turkey tendon}

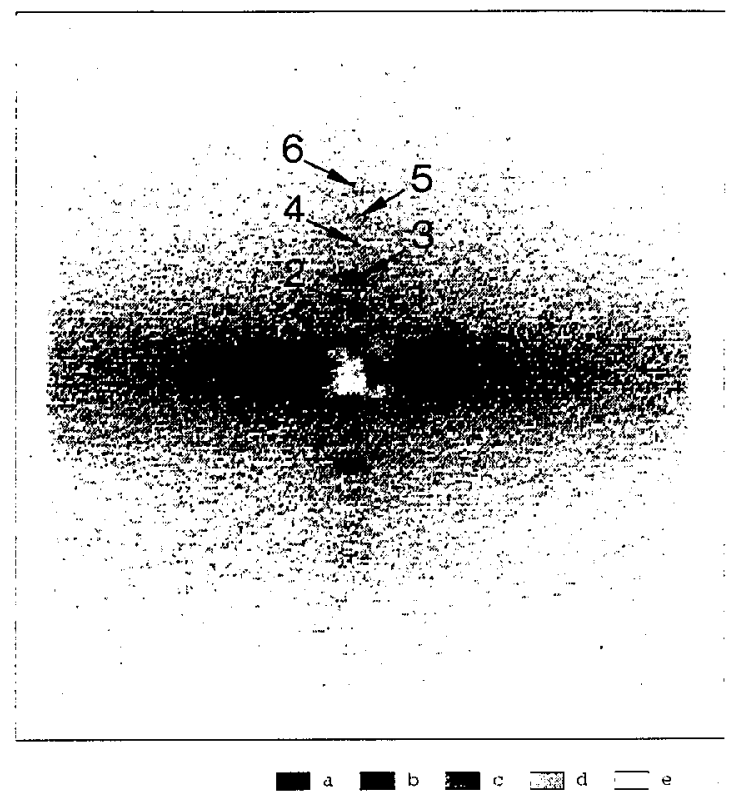

Fig. 13. Schematic representation of a mineralized collagen fibril (from [51]).

Fig. 14. X-ray scattering pattern from mineralized turkey leg tendon with the collagen fibrils oriented vertically (from [52]). The arrows indicate reflections from the axial period of the collagen fibril (see Fig.13).

and simultaneously the orientation of the mineral crystals. These are quite important parameters which are very difficult to obtain by other means [52].
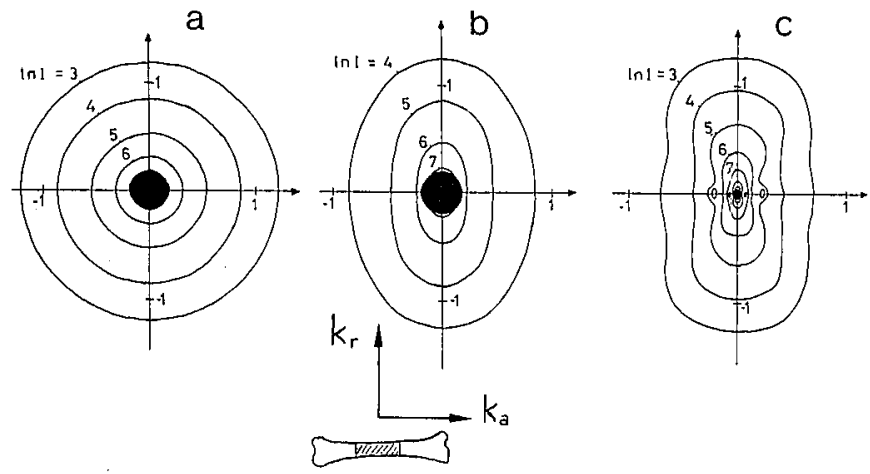

Fig. 15. Isointensity contours showing the small-angle X-ray scattering distribution from a mouse ulna (oriented as indicated). The horizontal and vertical scales are graduated in $\mathrm{nm}^{-1}$. (a) is an embryonic animal, (b) immediately after birth and (c) an adult individual. 
Figure 15, finally, shows the evolution of the crystal orientation with age for the ulna of a mouse [51]. For the adult animal (Fig. 15c), the crystals are oriented along the main axis of the bone, which give the bone a good mechanical stability. It is interesting to note that this preferential orientation does not exist in the bone of embroyos (Fig. 15a) and develops immediately after birth (Fig. 15b), as soon as the mechanical stress becomes important [51].

\section{Acknowledgements}

This work was supported in part by the Fonds zur Förderung der Wissenschaftlichen Forschung. The research on the microstructure of bone was supported partially by the Ludwig-Boltzmann Institut für Osteologie.

I am very grateful to my colleagues and co-authors, O. Blaschko, N. Fratzl-Zelman, M. Groschner, H.-G. Haubold, S. Klaumünzer, K. Klaushofer, K. Koller, F. Langmayr, J.L. Lebowitz, O. Paris, O. Penrose, H. Plenk, G. Vogl, Y. Yoshida, and I thank them for their collaboration and for many discussions.

\section{References}

[1] A. Gunier, Compt. Rend. 206, 1374 (1938).

[2] O. Kratky, G. Porod, J. Colloid Sci. 4, 35 (1949); G. Porod, Kolloid-Z. 124, 83 (1951); 125, 51, 109 (1952).

[3] A. Guinier, G. Fournet, Small-Angle Scattering of X-Rays, Wiley, London 1955.

[4] Small-Angle X-Ray Scaltering, Eds. O. Glatter, O. Kratky, Academic Press, London 1982.

[5] L.A. Feigin, D.I. Svergun, Structure Analysis by Small-Angle X-Ray and Neutron Scattering, Plenum Press, New York 1987.

[6] H.B. Stuhrmann, A. Miller, J. Appl. Crystallogr. 11, 325 (1978).

[7] H. Durchschlag, P. Zipper, R. Wilfing, G. Purr, J. Appl. Crystallogr. 24, 822 (1991).

[8] P. Laggner, M. Kriechbaum, G. Rapp, J. Appl. Crystallogr. 24, 836 (1991).

[9] J. Lowy, F.R. Poulsen, Biophys. J. 57, 977 (1990).

[10] T. Hashimoto, M. Takenaka, H. Jinnai, J. Appl. Crystallogr. 24, 457 (1991).

[11] W. Wilke, M. Bratrich, J. Appl. Crystallogr. 24, 645 (1991).

[12] T.P. Russel, in: Handbook of Synchrotron Radiation, Vol. 3, Eds. G.S. Brown, D.E. Moncton, Elsevier, Amsterdam 1991, p. 379.

[13] G. Kostorz, Mater. Sci. Forum 27/28, 325 (1988).

[14] G. Kostorz, J. Appl. Crystallogr. 24, 444 (1991).

[15] P.W. Schmidt, J. Appl. Crystallogr. 24, 414 (1991).

[16] A.J. Allen, J. Appl. Crystallogr. 24, 624 (1991).

[17] H.-G. Haubold, K. Gruenhagen, M. Wagner, H. Jungbluth, H. Heer, A. Pfeil, H. Rongen, G. Brandenberg, R. Moeller, J. Matzerath, P. Hiller, H. Halling, Rev. Sci. Instrum. 60, 1943 (1989).

[18] P. Fratzl, J.L. Lebowitz, J. Marro, M.H. Kalos, Acta Metall. 31, 1849 (1983).

[19] R.H. Ottewill, J. Appl. Crystallogr. 24, 436 (1991).

[20] O. Glatter, J. Appl. Crystallogr. 10, 415 (1977).

[21] D.I. Svergun, J. Appl. Crystallogr. 24, 485 (1991).

[22] H.B. Sthurmann, Adv. Polym. Sci. 67, 124 (1985). 
[23] H.B. Sthurmann, in: Topics in Current Chemistry, Vol. 145, Ed. E. Mandelkow, Springer, Berlin 1988, p. 151.

[24] D.H. Templeton, in: Handbook of Synchrotron Radiation, Vol. 3, Eds. G.S. Brown, D.E. Moncton, Elsevier, Amsterdam 1991, p. 201.

[25] J.P. Simon, O. Lyon, D. de Fontaine, J. Appl. Crystallogr. 18, 230 (1985).

[26] J.P. Simon, O. Lyon, Acta Metall. 7, 1727 (1989).

[27] Y. Waseda, in: Lecture Notes in Physics, Vol. 204, Eds. H. Araki, J. Ehlers, K. Hepp, R. Kippenhahn, H.A. Weidenmüller, J. Zittarz, Springer, Berlin 1984, p. 1.

[28] P. Fratzl, Y. Yoshida, G. Vogl, H.G. Haubold, to be published.

[29] P. Fratzl, J. Appl. Crystallogr. 24, 593 (1991).

[30] J.D. Gunton, M. San Miguel, P.S. Sahni, in: Phase Transitions and Critical Phenomena, Vol. 8, Eds. D. Domb, J.L. Lebowitz, Academic Press, London, p. 267.

[31] H. Furokawa, Adv. Phys. 34, 703 (1985).

[32] K.Binder, in: Materials Science and Technology, Phase Transformations in Materials, Vol. 5, Ed. P. Haasen, VCH Verlagsgesellschaft, Weinheim, p. 405.

[33] J.W. Cahn, Acta Metall. 9, 795 (1961).

[34] J.W. Cahn, J.E. Hilliard, J. Chem. Phys. 31, 688 (1959).

[35] H.E. Cook, Acta Metall. 18, 297 (1970).

[36] J.S. Langer, Acta Metall. 21, 1649 (1973).

[37] A. Chakrabarti, R. Toral, J.D. Gunton, Phys. Rev. B 39, 4386 (1989).

[38] T.M. Rogers, R.C. Desai, Phys. Rev. B 39, 11956 (1989).

[39] P. Fratzl, O. Blaschko, in: Dynamics of Ordering Processes in Condensed Matter, Eds. S. Komura, H. Furokawa, Plenum Press, New York 1988, p. 223.

[40] J. Marro, J.L. Lebowitz, M.H. Kalos, Phys. Rev. Lett. 43, 282 (1979).

[41] O. Blaschko, P. Fratzl, Phys. Rev. Lett. 51, 288 (1983).

[42] P. Fratzl, O. Blaschko, Phys. Rev. B 36, 2110 (1987).

[43] P. Fratzl, J.L.Lebowitz, Acta Metall. 37, 3245 (1989).

[44] P. Fratzl. J.L. Lebowitz, O. Penrose, J. Amar, Phys. Rev. B 44, 4794 (1991).

[45] F. Langmayr, P. Fratzl, G. Vogl, Acta Metall. Mater., in press.

[46] P. Fratzl, F. Langmayr, G. Vogl, W. Miekeley, Acta Metall. Mater. 39, 753 (1991).

[47] S. Messarolas, J.R. Schneider, R.J. Stewart, W. Zulehner, Nature 336, 364 (1988); S. Gupta, S. Messarolas, J.R. Schneider, R.J. Stewart, W. Zulehner, J. Appl. Crystallogr. 24, 576 (1991).

[48] O. Paris, M.Sc. thesis, University of Vienna, 1992; P. Fratzl, O. Paris, F. Langmayr, G. Vogl, H.G. Haubold, to be published.

[49] P. Fratzl, G. Vogl, S. Klaumünzer, J. Appl. Crystallogr. 24, 588 (1991).

[50] P. Fratzl, S. Klaumünzer, M. Rammensee, G. Vogl, Europhys. Lett. 11, 547 (1990).

[51] P. Fratzl, N. Fratzl-Zelman, K. Klaushofer, G. Vogl, K. Koller, Calcif. Tissue Int. 48, 407 (1991).

[52] P. Fratzl, M. Groschner, G. Vogl, H. Plenk Jr., J. Eschberger, N. Fratzl-Zelman, K. Koller, K. Klaushofer, J. Bone Miner. Res. 7, 329 (1992). 